\title{
Circuit
}

Musiques contemporaines

\section{Forum 2016 du NEM : un treizième rassemblement sous le signe de l'écoute active}

\section{Paul Bazin}

Volume 27, numéro 1, 2017

URI : https://id.erudit.org/iderudit/1039676ar

DOI : https://doi.org/10.7202/1039676ar

Aller au sommaire du numéro

Éditeur(s)

Les Presses de l’Université de Montréal

ISSN

1183-1693 (imprimé)

1488-9692 (numérique)

Découvrir la revue

Citer ce document

Bazin, P. (2017). Forum 2016 du NEM : un treizième rassemblement sous le signe de l'écoute active. Circuit, 27(1), 81-84. https://doi.org/10.7202/1039676ar d'utilisation que vous pouvez consulter en ligne.

https://apropos.erudit.org/fr/usagers/politique-dutilisation/ 


\section{Forum 2016 du NEM :}

\section{un treizième rassemblement \\ sous le signe de l'écoute active}

Paul Bazin

"I'm interested in dynamic systems of listening. " C'est dans ces mots que s'exprimait le compositeur canadien Stefan Maier lors de notre toute première rencontre. À elle seule, cette phrase résume assez bien une large part des conversations publiques qui eurent lieu dans le cadre de la $13^{\mathrm{e}}$ édition du Forum international des jeunes compositeurs du Nouvel Ensemble Moderne (NEM), tenue à Montréal du 2 au 25 novembre dernier. Posant d'emblée la nécessité d'une écoute active, cette affirmation nous parle à la fois de l'écoute de l'auditeur et de celle du compositeur, pratique nécessaire tant à l'élaboration qu'à la réception d'une œuvre.

Au moment de m’offrir cette réflexion, Maier me proposait en fait sa propre réponse à une question formulée par John Rea, et autour de laquelle devaient s'articuler les différentes discussions publiques du Forum que j’ai animées ${ }^{1}$. Portant sur la projection d'un auditeur virtuel par le compositeur, elle se lisait comme suit: «Le compositeur écrit-il pour un auditeur idéal, pour quelqu'un de réceptif au changement personnel, qui résiste à l'esclavage auditif, qui n'est pas dupe de la vocifération inutile? » Les sept discussions publiques inscrites à l'horaire du Forum, accueillies par la
Faculté de musique de l'Université de Montréal et le Centre de musique canadienne au Québec, avaient pour principal objectif de générer un grand mouvement de réflexion autour de cette question. Dans cette perspective, les huit jeunes compositeurs appartenant à la plus jeune génération - tous sont nés entre 1988 et 1997 - eurent tour à tour l'occasion de présenter leur parcours, leur démarche et leur musique, les uns se confrontant bientôt aux idées des autres.

Toutes ces rencontres furent autant d'occasions de plonger dans des mondes sonores situés aux antipodes du spectre stylistique: des structures mathématiques de Sebastian Dumitrescu (Roumanie), le public fut invité à passer aux sons concrets tirés de l'expérience d'enregistrement en studio de Daniel Miller (É.-U.), à s'interroger sur le caractère engagé des titres de Sky Macklay (É.-U.) et à apprécier les multiples perspectives d'écoute offertes dans la musique de Quentin Lauvray (France). Difficile, encore, de ne pas mentionner l'écologie acoustique des pièces de Maier, aux sonorités abrasives, ou encore la densité de certaines œuvres d'Alican Çamcı (Turquie) et de William Kuo (Canada). A priori, les intersections semblaient rares entre tous ces musiciens, bien que certains points 
de convergences aient fini par se révéler. En voici quelques exemples.

D'abord, chez la majorité des huit compositeurs, l'abstraction sonore domine. Inutile, en conséquence, de se perdre à la recherche de significations précises dans leurs musiques. En ce sens, le travail de Lauvray donna matière à réflexion. Lun de ses principes fondamentaux étant la combinaison de strates sonores contrastantes, il interroge la capacité de l'auditeur à s'engager sur plusieurs niveaux d'écoute à la fois. À ce sujet, Lauvray confie qu'il voit dans sa musique un jeu de perception, et spécifie que l'auditeur est libre de porter attention à l'aspect qu'il souhaite. De ce fait, l'œuvre devient en quelque sorte « ouverte ", puisqu'on est invités à y naviguer et à inventer chaque fois de nouvelles trajectoires d'audition.

Ensuite, l'impact d'Helmut Lachenmann sur l'imaginaire sonore de nos contemporains est peut-être l'un des sujets qui fédérèrent le plus les participants de l'événement. Tant à l'occasion des multiples discussions que dans les œuvres écrites à l'intention du Forum, l'influence de ce compositeur ne pouvait manquer d'attirer l'attention. Qu'on pense aux œuvres de Kuo (The Weight of Transparency), de Çamcı (Close-up), de Sheets (From Cairn Terrain) ou de Maier (Thicket III), la présence récurrente de détails infimes dans le son ne manquait de rappeler l'impulsion nouvelle que le maître allemand a donnée à la réflexion sur les liens entre le son musical traditionnel et le bruit.

Enfin, l'un des aspects récurrents de toutes les conversations que j'ai eues avec les compositeurs tient dans le peu d'intérêt - ou d'affinité - qu'ils ont montré à l'endroit de la question formulée par Rea. L'accueil qui y fut réservé s'est en effet avéré mitigé, les discussions les plus riches glissant d'ordinaire vers des préoccupations d'ordres tout autres. On eut pourtant droit à quelques réponses, parfois brèves et directes: Kuo avançait être lui-même son auditeur idéal, consi- quelque auditeur entendant la musique de la façon que lui-même l'entend, alors que Çamcı soutenait plutôt que l'auditeur idéal n'existe tout simplement pas, les caractéristiques d'une musique supposant en soi, au demeurant, un certain type d'auditeur; encore, Daniel Miller voyait pour sa part l'écoute interne du compositeur comme une phase de pré-écoute, qui n’a à voir qu'avec sa seule démarche. De leur côté, Macklay et Dumitrescu plaidaient enfin pour une musique qui, bien que pouvant être complexe dans sa facture, appelle d'abord une réponse plus épidermique qu'intellectuelle chez l'auditeur, et donc, peut-être, plus spontanée.

Dans un tout autre ordre d'idée, j’avoue avoir été agréablement surpris de retrouver, dans les propos de ces musiciens qui proviennent d'un peu partout de par le monde, un vif écho aux préoccupations disons «locales»- exprimées dans les récentes prises de parole de Symon Henry et de Gabriel Ledoux². Àce propos, Stefan Maier est apparu comme un individu particulièrement articulé, animé par la volonté de déconstruire les images figées du milieu musical dans lequel nous œuvrons. Plaidant sans modération contre la sclérose qu'engendre l'institutionnalisation de la musique de création, il abonda dans le même sens que Dumitrescu, insistant sur le fait que les nouvelles générations de mélomanes, plus mobiles que jamais, peuvent, sans s'en formaliser, assister un soir à un show de musique métal et à un concert de musique moderne le lendemain. Les frontières entre les genres musicaux s'avèrent poreuses pour ces musiciens qui agissent tant comme compositeurs que comme interprètes, et qui naviguent régulièrement entre les sphères classique, métal et jazz, n’hésitant pas jeter des passerelles entre elles ${ }^{3}$. Au fil de ces propos, soulignons à quel point la vitrine offerte par le Forum du NEM s'avérait opportune à quiconque s'intéresse aux intérêts de la plus jeune génération de créateurs.

Présentées à l'occasion des deux concerts de clôture du Forum, les œuvres des huit compositeurs témoi- 
gnaient évidemment de cette vaste palette d'intérêts et de considérations. Les contraintes imposées aux compositeurs en vue de l'écriture de leurs pièces ayant été peu nombreuses, on eut droit à des œuvres fort différentes les unes des autres. Soignées tant dans leurs factures respectives que dans la justesse des exécutions qu'en offrit le NEM, les pièces me semblaient se poser comme autant de regards sur le milieu actuel de la musique de création et ses multiples facettes. Les deux premiers prix du Forum, décernés respectivement à Zachary Sheets et à Quentin Lauvray, furent attribués pour des œuvres bien écrites et s'inscrivant dans une assez large mouvance de musique sérieuse qu'on reconnaît comme réfléchie, construite et maîtrisée. De la musique contemporaine en bonne et due forme, en somme - c'était peu étonnant qu'on leur octroie les deux premiers prix -, qui m’a semblée légèrement prudente et à laquelle j'aurais personnellement souhaité voir s'ajouter un brin de risque, d'audace et, peut-être même, d'irrévérence. Çamcı et Dumitrescu, à qui l'on a remis le troisième prix ex aequo, proposaient des pièces très différentes, l'écologie délicate et l'utilisation d'objets concrets par le Turc rompant avec les aspects plus immédiatement audibles de la musique de son confrère Roumain. Il faut souligner que les complexités mathématiques de la musique de Dumitrescu ne menaient en rien à une écoute laborieuse ou cérébrale, que, bien au contraire, les intersections entre les lignes musicales auxquelles étaient confiées les transcriptions des algorithmes rendaient aisément audible cette démarche pourtant abstraite, par ailleurs doublée d'un très beau travail sur les combinaisons et les successions de timbres venant soutenir une trame harmonique aux couleurs étonnantes. À mon sens, l'œuvre de Dumitrescu constitue l'une des propositions les plus originales du Forum, aux côtés des pièces de Macklay et de Maier. Le titre de l'Américaine, Microvariations, est l'une des trop rares pièces qu'il m'ait été donnée d'entendre qui parvienne réellement à introduire l'humour en musique sans tomber dans la niaiserie. N'hésitant pas à faire usage d'éléments tirés de la syntaxe tonale, cette pièce enchaîne à un rythme effréné cadences et marches d'harmonie tout en les passant à travers le filtre d'un orchestre scindé en deux, dont les deux groupes instrumentaux étaient accordés à distance d'un quart de ton l'un de l'autre.

Pour conclure, j’aimerais formuler un plaidoyer en faveur de la pièce de Maier. Tirant profit de toutes les sonorités rendues possibles par des objets qu'on ne qualifie pas, d'ordinaire, de «musicaux» (ratchets, feuilles de styromousse, vibrateur, etc.), le compositeur a conçu un environnement sonore à mi-chemin entre la trame bruitiste et l'œuvre électroacoustique traditionnelle. Suite à l'exécution de sa pièce en concert, on a fait grand cas de cet instrumentarium qui n'a pourtant rien d'extraordinaire, et qu'on pourrait sans contorsion rapprocher de la musique concrète et du solfège des objets sonores, développés il y a quelques décennies déjà par Pierre Schaeffer. Conviant l'auditeur à une écoute rapprochée de flexions sonores infimes, cette musique s'inscrit dans une mouvance qui croît de plus en plus, et qui inclue notamment les Toposition(s) de Charles-Antoine Fréchette ou certaines des œuvres de James O'Callaghan, pour ne nommer qu'eux, et qui appartiennent de surcroît à la même génération que Maier. Il me semble en effet qu'on a depuis longtemps démontré que nos oreilles sont capables d'identifier les nuances sonores les plus fines. Si de telles œuvres bousculent encore, c'est peut-être qu'elles interrogent notre ouverture vis-à-vis des prospections sonores les moins conventionnelles. Le Forum ayant ceci de formidable qu'il cède la parole aux plus jeunes générations, pourquoi ne pas nous aussi jouer le jeu, en tant qu'auditeur, et tendre l'oreille? 
1. Le texte complet de Rea, comprenant la question soumise aux compositeurs, a été imprimé dans le document produit à l'occasion du Forum. Il est possible de se procurer ce document auprès du Nouvel Ensemble Moderne.

2. Voir Symon Henry (2016), "Entendu dans Cette Ville Étrange: "Fuck toute!" - une génération de compositeur.e.s décomplexe sa marge", Circuit, musiques contemporaines, vol. 26, $\mathrm{n}^{\circ} 2$ 2, p. 77-81;

Gabriel Ledoux, "SMCQ et NEM : les systèmes politiques oligarchiques et leurs conséquences sur la représentativité de l'art subventionné",
$<$ http://art-raffine.tumblr.com> (consulté le 21 décembre 2016). Pour une réponse à ces deux textes, voir Michel Gonneville, "Écoutons-les: réflexions sur deux coups de gueule...", <www.cettevilleetrange.org/ ecoutons-les> (consulté le 21 décembre 2016).

3. Au moins quatre des huit compositeurs du Forum pratiquent la musique à titre d'interprètes: Stefan Maier est interprète de musique électronique, Sky Macklay hautboïste, Zachary Sheets est flûtiste et Sebastian Dumitrescu joue de la basse électrique.

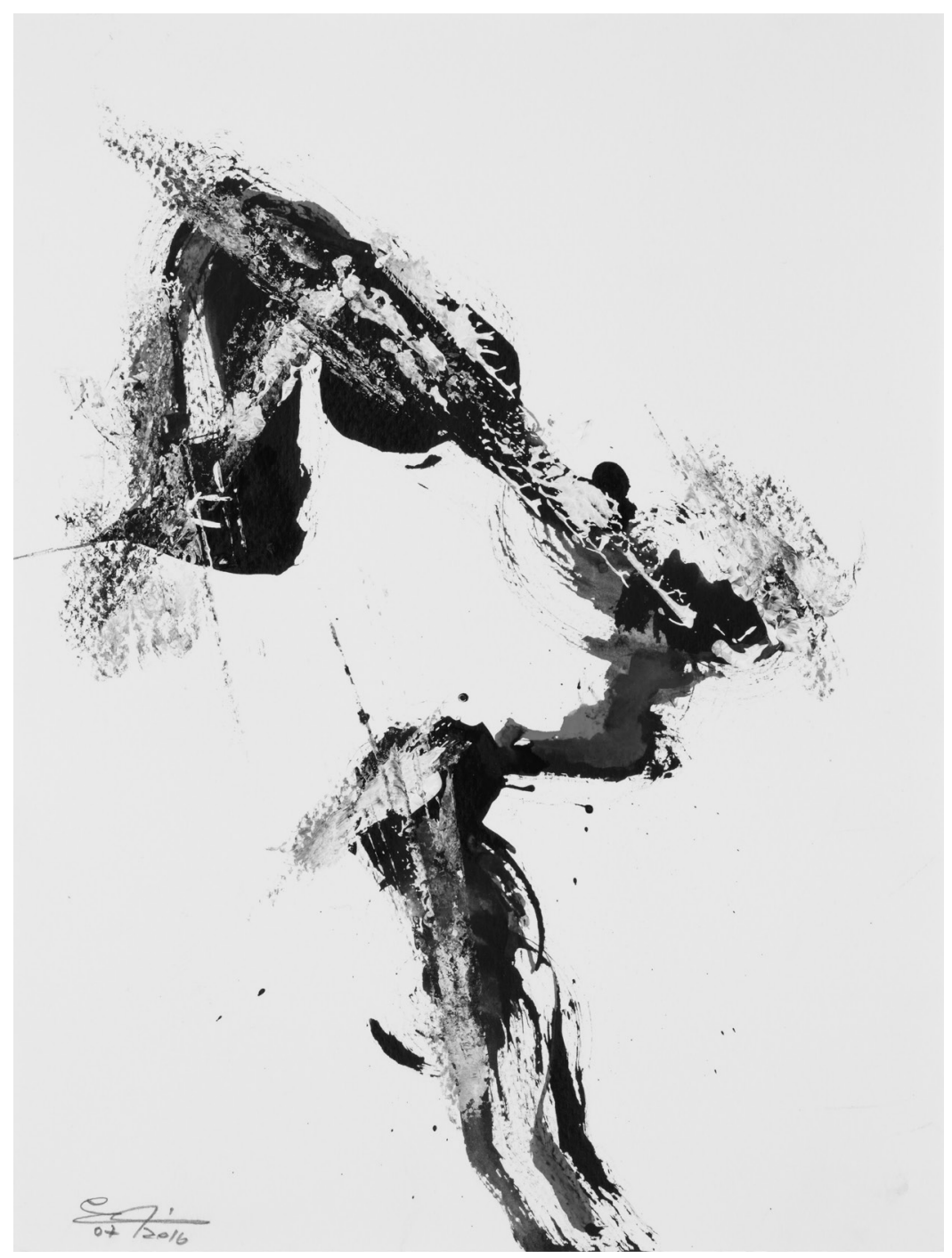

Rita Ezrati, Arabesque, 2016. Techniques mixtes sur papier, $32 \times 24 \mathrm{~cm}$. Photo: Richard-Max Tremblay. 